Supporting Information for

\title{
Perturbation Electrochemiluminescence Imaging to Observe the Fluctuation of Charge-Transfer Resistance in Individual Graphene Microsheets with Redox-Induced Defects
}

Hui Zhu, Rong Jin, Dechen Jiang, "Jun-Jie Zhu,

†State Key Laboratory of Analytical Chemistry for Life Science, School of Chemistry and Chemical Engineering, Nanjing University, Nanjing, 210023, P. R. China

\section{Corresponding Author}

*Email: dechenjiang@nju.edu.cn (D.J); jjzhu@nju.edu.cn (J.-J.Z) 


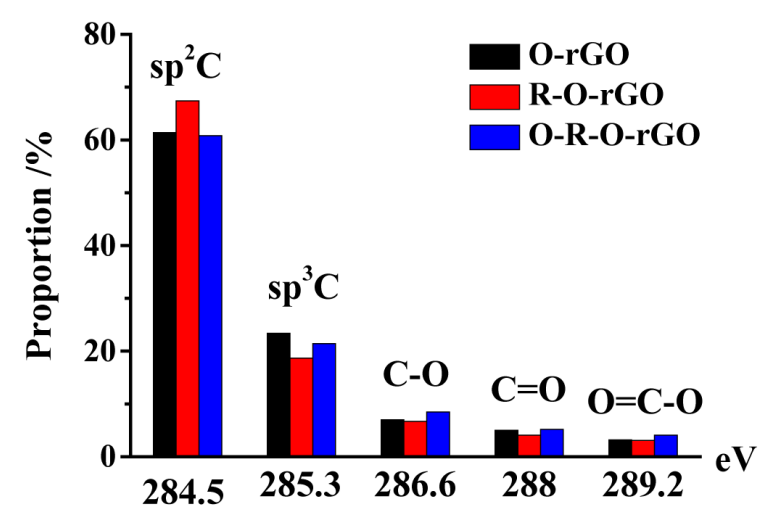

Figure S1. Analysis result of defects and the surface oxygen functional groups from rGO microsheets during this redox process. O-rGO: rGO after the electrochemical oxidation; RO-rGO: rGO after the oxidation and reduction; O-R-O-rGO: rGO after the oxidation, reduction and the oxidation.

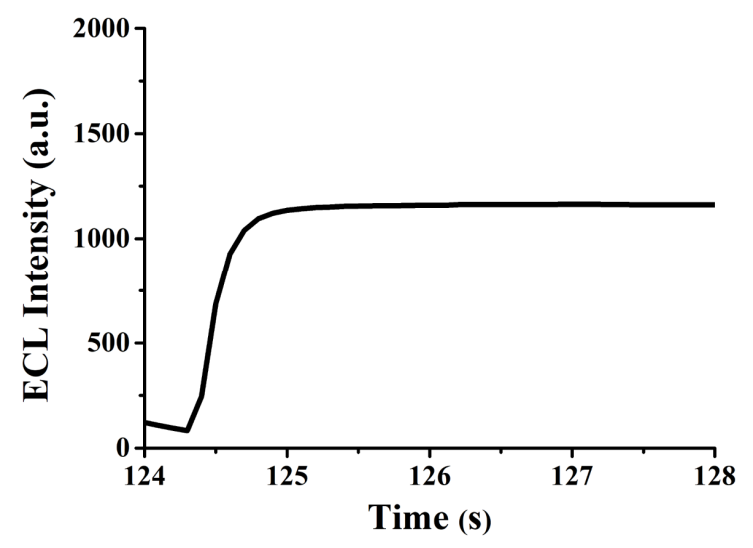

Figure S2. ECL trace of ITO slide during the electrochemical reduction under $0 \mathrm{~V}$ for $1 \mathrm{~s}$, and then, the oxidation under $0.8 \mathrm{~V}$ for $4 \mathrm{~s}$. 


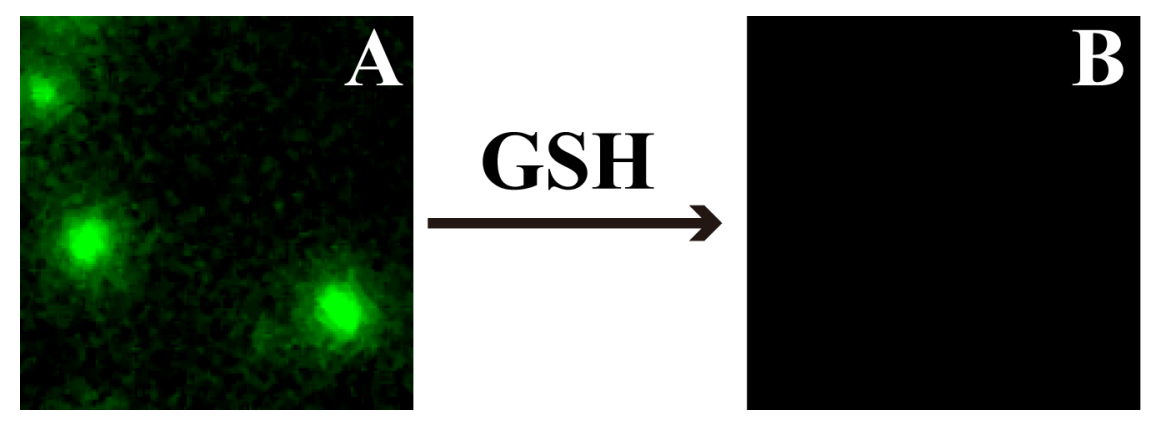

Figure S3. The ECL images of (A) individual rGO microsheets, B) individual rGO microsheets in presence of the radical scavenger (GSH). The solution is $1 \mathrm{X}$ PBS with 200 $\mu \mathrm{M}$ L012.

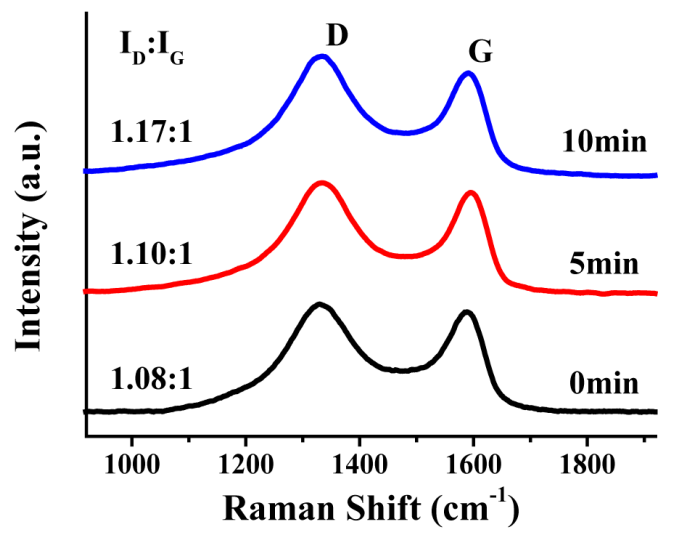

Figure S4. Raman spectrums of rGO microsheets after the electrochemical oxidation under $0.8 \mathrm{~V}$ for 0,5 and $10 \mathrm{~min}$, respectively. 


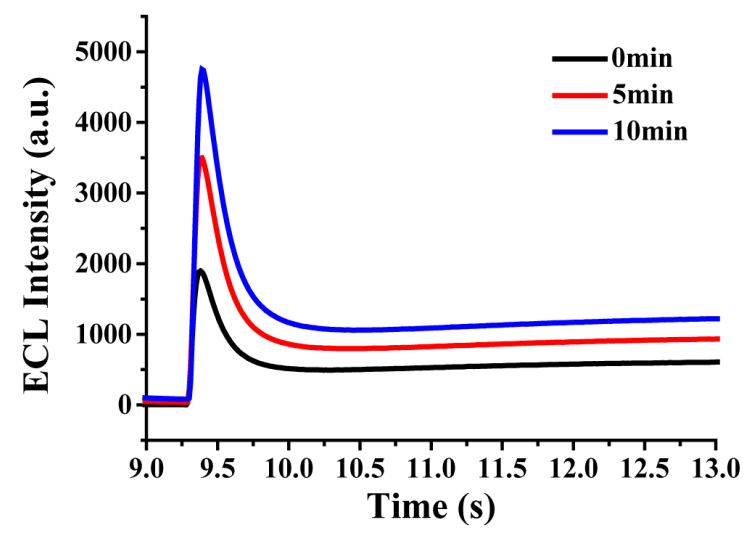

Figure S5. ECL intensities of rGO microsheets after the electrochemical oxidation under $0.8 \mathrm{~V}$ for 0,5 and $10 \mathrm{~min}$, respectively.

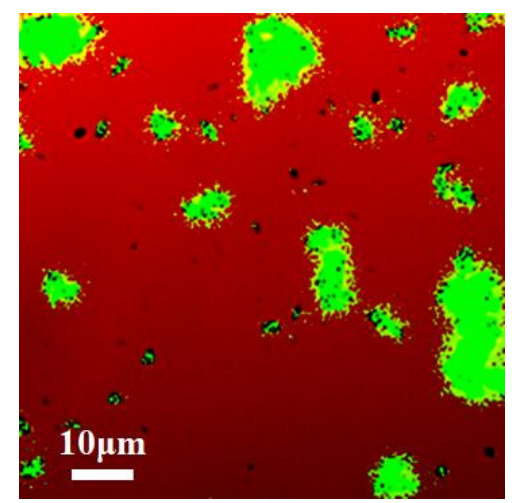

Figure S6. The pseudo-color overlapping image from the bright-field (Figure 4A) and ECL (Figure 4B) images. The yellow part exhibits the extension range of ECL illumination at individual rGO microsheets. 


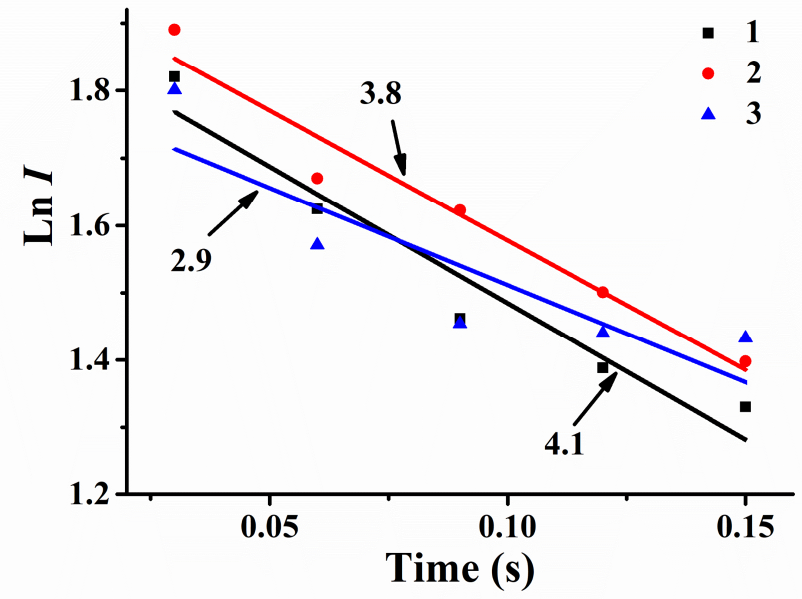

Figure S7. The correlation between Ln I collected from three rGO microsheets (labelled in Figure 5A) and the time applied with high potential. The lines are the fitting curves.

Table S1. The percent composition (\%) for $\mathrm{sp}^{2}, \mathrm{sp}^{3}, \mathrm{C}-\mathrm{O}, \mathrm{C}=\mathrm{O}$ and $\mathrm{O}=\mathrm{C}-\mathrm{O}$ in the samples of O-rGO, R-O-rGO and O-R-O-rGO.

\begin{tabular}{llllll}
\hline Samples & $\mathbf{s p}^{\mathbf{2}}$ & $\mathbf{s p}^{\mathbf{3}}$ & $\mathbf{C - O}$ & $\mathbf{C}=\mathbf{O}$ & $\mathbf{O}=\mathbf{C}-\mathbf{O}$ \\
\hline O-rGO & 61.4 & 23.4 & 7.0 & 5.0 & 3.2 \\
R-O-rGO & 67.4 & 18.7 & 6.7 & 4.1 & 3.1 \\
O-R-O-rGO & 60.8 & 21.4 & 8.5 & 5.2 & 4.1 \\
\hline
\end{tabular}


Table S2. The corresponding values of $\mathrm{a}, \mathrm{b}$ and $\mathrm{c}$ in the samples of O-rGO, R-O-rGO and O-R-O-rGO.

\begin{tabular}{llll}
\hline Samples & O-rGO & R-O-rGO & O-R-O-rGO \\
\hline $\mathrm{a}\left(\mathrm{R}_{\mathrm{s}}\right)(\Omega)$ & 181.41 & 159.75 & 181.15 \\
$\mathrm{~b}\left(\mathrm{R}_{\mathrm{ct}}\right)(\Omega)$ & 21256 & 8899 & 27704 \\
$\mathrm{c}\left(\mathrm{C}_{\mathrm{d}}\right)(\mathrm{F})$ & $4.00 \mathrm{E}-5$ & $2.18 \mathrm{E}-5$ & $4.16 \mathrm{E}-5$ \\
\hline
\end{tabular}

Table S3. The ECL intensity collected from 11 rGO microsheets (No.1 No. 11) at different time, and the calculated value of $\alpha$ from these microsheets.

\begin{tabular}{|c|c|c|c|c|c|c|c|c|c|c|c|}
\hline Time/s & & & & & Relati & ve ECL In & ensity & & & & \\
\hline & No. 1 & No. 2 & No. 3 & No.4 & No. 5 & No.6 & No. 7 & No.8 & No.9 & No. 10 & No.11 \\
\hline 0.03 & 6.7246 & 6.1731 & 8.7166 & 6.6212 & 6.053 & 5.18056 & 6.404 & 6.4848 & 7.6905 & 4.9375 & 6.425 \\
\hline 0.06 & 5.5585 & 5.0769 & 5.6478 & 5.3131 & 4.8106 & 3.61111 & 5.6768 & 4.9798 & 5.381 & 3.97917 & 4.825 \\
\hline 0.09 & 4.8677 & 4.3141 & 5.1336 & 5.0657 & 4.2803 & 3.58333 & 5.0909 & 4.6465 & 4.3095 & 3.83333 & 3.975 \\
\hline 0.12 & 4.4646 & 4.0064 & 4.3401 & 4.4848 & 4.2197 & 3.29167 & 4.0303 & 4.6566 & 4.5476 & 2.5625 & 3.625 \\
\hline 0.15 & 4.1846 & 3.7821 & 4.498 & 4.0455 & 4.1894 & 3.13889 & 3.7677 & 4.3939 & 3.9286 & 3.08333 & 3.575 \\
\hline$\alpha($-intercept $)$ & 3.89 & 4.06 & 5.29 & 3.85 & 2.89 & 3.65 & 4.68 & 2.82 & 5.04 & 4.6 & 4.86 \\
\hline
\end{tabular}

